\title{
Eosinophilic Gastroenteritis: Review of a Rare and Treatable Disease of the Gastrointestinal Tract
}

\author{
Amit Mori $^{a} \quad$ Chijioke Enweluzo $^{a} \quad$ David Grier $^{b} \quad$ Madhu Badireddy $^{a}$ \\ ${ }^{\mathrm{a}}$ Section on Hospital Medicine, Department of Internal Medicine, and ${ }^{\mathrm{b}}$ Department of \\ Pathology, Wake Forest School of Medicine, Winston Salem, N.C., USA
}

\section{Key Words}

Eosinophilic gastroenteritis - Desensitization - Samter's triad · Infiltration · Erythematous · Mucosal

\begin{abstract}
Eosinophilic gastroenteritis is a rare disease of the gastrointestinal tract characterized by crampy abdominal pain, nausea, vomiting, diarrhea, gastrointestinal bleeding, and weight loss associated with peripheral eosinophilia leading to eosinophilic infiltrates in stomach and intestine, usually in a patient with a prior history of atopy. In this article, we describe our encounter with a 59-year-old female presenting with severe abdominal pain, nausea, vomiting, and weight loss with an extensive evaluation including an upper endoscopy with biopsies resulting in a diagnosis of eosinophilic gastroenteritis. The patient was eventually treated with oral prednisone for three weeks with complete resolution of her symptoms.
\end{abstract}

(C) 2013 S. Karger AG, Basel

\section{Introduction}

Eosinophilic gastroenteritis (EGE) is a rare gastrointestinal disease characterized by crampy generalized abdominal pain, nausea, vomiting, diarrhea, gastrointestinal bleeding and weight loss or various combinations of the above symptoms. The etiology of the disease remains unknown. It is usually encountered in patients with a history of atopy. The disease may involve any part of the gastrointestinal tract, but the stomach and the small intestine are the most common sites involved. Isolated eosinophilic esophagitis or colitis may occur, but they are not discussed in this article. EGE is associated with peripheral eosinophilia leading to eosinophilic infiltrates in the different layers of the stomach and intestine. Clinical 
presentation may vary depending on sites and depth of involvement of the gastrointestinal tract. Imaging has very little role in supporting the diagnosis, and therefore a high degree of clinical suspicion is required. The diagnosis is usually confirmed by an upper endoscopy and microscopy that shows more than 20 eosinophils per high-power field in the association with peripheral eosinophilia and the absence of secondary cause of eosinophilia. Although the disease may relapse in some cases after steroid therapy, most patients respond to steroid therapy with full resolution of symptoms. Our experience with this patient demonstrates that early diagnosis will lead to full treatment, thereby preventing unnecessary interventions, and help improve the patient's overall health.

\section{Clinical Course}

We present a 59-year-old Caucasian female with a past medical history of asthma, nasal polyps, aspirin sensitivity (Samter's triad), sarcoidosis and pancreatitis admitted with 2 weeks of abdominal pain, nausea, and intractable vomiting, diarrhea and weight loss. A few weeks prior to presentation, she underwent aspirin desensitization with about 4 weeks of escalating aspirin dose $(81,162,325$ and $650 \mathrm{mg}$, escalating weekly). During the fourth week of desensitization, she took aspirin $650 \mathrm{mg}$ twice a day for 1 day and developed severe abdominal pain, nausea, vomiting and inability to tolerate any kind of diet, and was subsequently admitted for further investigation.

She was managed for presumed acute aspirin-induced pancreatitis with lipase of $353 \mathrm{U} / \mathrm{l}$ and mildly dilated biliary tree on abdominal ultrasound. She was noted to have hives and her aspirin was stopped. This was also associated with a mild elevation of her absolute eosinophil count to 3,600 cells/ $\mu$ l even after stopping the offending drug, normocytic mild anemia with $\mathrm{Hb}$ of $11.1 \mathrm{~g} / \mathrm{dl}$, albumin of $2.3 \mathrm{~g} / \mathrm{dl}$, total bilirubin 1.4 and normal AST and ALT (table 1). Gastroenterology was consulted and she underwent abdominal CT, MRCP, upper gastrointestinal series and gastric emptying study, which were all unremarkable except for mild delayed gastric emptying. The patient clinically improved and she was then discharged with plans for outpatient evaluation of her peripheral eosinophilia.

A week later, the patient developed severe abdominal pain, nausea, vomiting and diarrhea again and had to return to the emergency room. She also reported weight loss of about $7 \mathrm{~kg}$ in the last 4 weeks. During this admission, her vital signs were: temperature $98.9^{\circ} \mathrm{F}$, heart rate 103 beats/min, blood pressure 139/92 mm Hg and oxygen saturation 95\% on room air. Her physical exam was only significant for cachexia and epigastric tenderness without rebound or guarding. Pertinent labs on admission showed: white blood cell count $13.8 \times 10^{3}$, hemoglobin $11.6 \mathrm{~g} / \mathrm{dl}, \mathrm{MCV} 88.9 \mathrm{fl}$, absolute eosinophil count of 13,600 cells/ $\mu \mathrm{l}$ (72\%), albumin 2.0, normal liver function tests and normal lipase level (table 1). Gastroenterology was again consulted. She underwent an EGD that showed mild diffuse gastritis in the entire stomach and moderate to severe duodenitis with erythematous mucosa and edematous villi. Multiple biopsy specimens showed moderate eosinophilic infiltration in the stomach mucosa with similar findings in the duodenal mucosa. Duodenal biopsy showed up to 84 eosinophils per high-power field (normal $<10$ eosinophils), which was highly indicative of EGE (fig. 1). No invasion to the muscular or subserosal layers was seen. During the work-up for hypereosinophilia she was found to have elevated IgG4 levels, and hematology as well as rheumatology consults were obtained for evaluation of Churg-Strauss vasculitis and hematologic malignancy. She underwent a bone marrow biopsy which showed many atypical eosinophils, most likely driven by a secondary process. However, oncology recommended sending out chromosomal testing to make sure none of the cells showed signs 
Mori et al.: Eosinophilic Gastroenteritis: Review of a Rare and Treatable Disease of the Gastrointestinal Tract

consistent with leukemia, and the work-up for this was subsequently negative including BCR-ABL mutation. Hypereosinophilic syndrome was deemed unlikely because of acute to subacute presentation and older age of the patient. Stool studies for ova and parasites were negative. Serology for Strongyloides was also negative. The diagnosis of hypereosinophilia leading to acute EGE was made.

The patient was started on prednisone $30 \mathrm{mg}$ daily. After an initial dose of steroids, she clinically improved significantly, her abdominal pain, nausea and vomiting improved, and she tolerated her diet. Her absolute eosinophil count dropped to $<1,000$ and she was discharged on prednisone $30 \mathrm{mg}$ daily for 2 weeks with subsequent tapering. She was followed up as an outpatient after 3 weeks with complete resolution of her symptoms and weight gain of about $3 \mathrm{~kg}$, normal eosinophil count and albumin of $3.0 \mathrm{~g} / \mathrm{dl}$. She thereafter remained symptom-free for 4 months.

\section{Discussion}

EGE is a rare disease of the gastrointestinal tract that is characterized by crampy abdominal pain, nausea, vomiting, diarrhea, gastrointestinal bleeding and weight loss associated with peripheral eosinophilia leading to eosinophilic infiltrates in the stomach and intestine, usually in a patient with a prior history of atopy $[1,2]$. Its incidence is difficult to estimate owing to the rarity of the disease. Since the very first description of this disease by Kaijser in 1937, more than 280 cases have been reported in the medical literature. This disease affects both adults and children. There is a slight male predominance, and it is reported to be more common in Caucasians. In 1970, Klein et al. [3] classified the disease according to the anatomic location of eosinophilic infiltration in different layers of the intestinal wall - the mucosal, muscularis and subserosal layers. They defined three patterns of disease manifestation based on symptoms and location as well as on depth of involvement by eosinophilic infiltration. Presentation may vary depending on location as well as depth and extent of bowel wall involvement and usually runs a chronic relapsing course. It can be classified into mucosal, muscular and serosal types based on the depth of involvement. Any part of the gastrointestinal tract can be involved, however the stomach is the organ most commonly affected, followed by the small intestine and the colon $[4,5]$. Isolated pancreatobiliary system involvement has also been reported [6]. The etiology and pathogenesis are not well understood and mostly based on case reports. Many patients have a history of seasonal allergy, atopy, food allergies, asthma and elevated serum IgE levels that may strongly suggest the role of hypersensitivity reactions in the pathogenesis of EGE [7]. Small amount of eosinophils are normally present in the mucosa as a host defense mechanism, but their presence in the deeper layers is almost always abnormal. A review of literature also suggests a role of various cytokines (interleukin 3, interleukin 4, granulocyte macrophage colonystimulating factor) and eotaxin, which are produced by eosinophils. It is also postulated that food allergens may cross the mucosa and play a role in local recruitment of eosinophils $[8,9]$.

Patients may have various clinical symptoms based on location and depth of involvement. The mucosal form, which is more common and seen in about $25-100 \%$ of cases, usually presents as abdominal pain, nausea, vomiting, dyspepsia, diarrhea, malabsorption, gastrointestinal hemorrhage, protein-losing enteropathy and weight loss. The muscularis form (seen in about 10-60\% of cases) usually follows the clinical picture of obstructive symptoms due to pyloric stenosis, gastric outlet obstruction and, rarely, intussusception. The subserosal form, which is less common, usually presents with significant bloating, exuda- 
Mori et al.: Eosinophilic Gastroenteritis: Review of a Rare and Treatable Disease of the Gastrointestinal Tract

tive ascites and a comparably higher number of peripheral eosinophilia than other forms [10-12].

Laboratory findings that support the diagnosis of EGE include, but are not limited to, peripheral eosinophilia (ranging from 5 to $70 \%$ ), hypoalbuminemia, abnormal D-xylose test, increased fecal fat, iron deficiency anemia, abnormal liver function tests, prolonged prothrombin time and elevated serum IgE levels. The erythrocyte sedimentation rate is rarely elevated $[3,12]$. Barium studies are usually abnormal in the muscularis form and may show luminal narrowing and irregularities in the distal antrum and the proximal small bowel. Diagnosis required high clinical suspicion and upper endoscopies with multiple biopsies from normal- and abnormal-appearing mucosa (in the mucosal form mainly), although laparoscopic full-thickness biopsies may be necessary in the muscularis and subserosal forms of EGE (fig. 1).

The differential diagnoses, among many other possibilities, include intestinal parasitic infections (can be excluded by stool studies), primary hypereosinophilic syndrome (persistent marked eosinophilia for 6 months or more, rarely involving the gastrointestinal system), malignancies (gastric cancer, lymphoma - excluded by laboratory, immunohistochemistry, biopsy) and the vasculitic phase of Churg-Strauss syndrome [12-14]. The role of imaging in the diagnosis of EGE is very limited because radiological findings are nonspecific and absent in half of the patients. Endoscopy usually reveals erythematous, friable, sometimes nodular mucosa and rarely ulceration in the stomach. There can also be diffuse enteritis with flattening of the mucosal surface in the proximal small intestine. Rarely, a large ulcerative mass with obstruction can be seen. The most common histologic findings are crypt hyperplasia and eosinophilic infiltration in the lamina propria. Microscopy usually shows an eosinophil count of 20 or more per high-power field (our patient had 78 eosinophils per high-power field) [15].

Treatment is based on the severity of symptoms. Mild symptoms are managed with careful search of inciting food allergens, medication review and avoiding them if found. Most patients present with moderate to severe symptoms. Corticosteroids are the mainstay of therapy in these patients. The usual dose of prednisone is $20-40 \mathrm{mg}$ daily for 2 weeks with tapering thereafter. A vast majority of patients improve with this therapy and do not require further treatment. However, relapse can occur and is treated with long-term, low-dose steroids (prednisone 5-10 mg daily). Among other medications, mast cell stabilizers, antihistamines and selective leukotriene receptor antagonist (montelukast) have shown positive results in some patients $[3,12,16,17]$. The presentation of our case demonstrates that although EGE is a rare disease, a high level of clinical suspicion, especially in patients with a history of allergy and peripheral eosinophilia, will aid in early diagnosis and prompt treatment. On the other hand, if left untreated or missed altogether, it may lead to further involvement of deep layers of the gastrointestinal system wall, causing further complications that may require invasive measures, thereby affecting quality of life.

\section{Disclosure Statement}

The authors declare no conflict of interest. There was no grant support. 
Mori et al.: Eosinophilic Gastroenteritis: Review of a Rare and Treatable Disease of the Gastrointestinal Tract

\section{References}

1 Kaijser R: Zur Kenntnis der allergischen Affektionen des Verdauungskanals vom Standpunkt des Chirurgen aus. Arch Klin Chir 1937;188:36-64.

2 Whitaker I, Gulati A, McDaid J, Bugajska-Carr U, Arends M: Eosinophilic gastroenteritis presenting as obstructive jaundice. Eur J Gastroenterol Hepatol 2004;16:407-409.

-3 Klein N, Hargrove R, Sleisenger M, Jeffries G: Eosinophilic gastroenteritis. Medicine (Baltimore) 1970;49: 299-319.

4 Treiber GG, Weidner S: Eosinophilic gastroenteritis. Clin Gastroenterol Hepatol 2007;5:e16.

-5 Naylor A: Eosinophilic gastroenteritis. Scott Med J 1990;35:163-165.

-6 Jimenez-Saenz M, Villar-Rodriguez J, Torres Y, Carmona I, Salas-Herrero E, Gonzalez-Vilches J, HerreriasGutierrez J: Biliary tract disease: a rare manifestation of eosinophilic gastroenteritis. Dig Dis Sci 2003;48: 624-627.

7 Oyaizu N, Uemura Y, Izumi H, Morii S, Nishi M, Hioki K: Eosinophilic gastroenteritis. Immunohistochemical evidence for IgE mast-cell mediated allergy. Acta Pathol Jpn 1985;35:759-766.

-8 Desreumaux P, Bloget F, Seguy D, Capron M, Cortot A, Colombel J, Janin A: Interleukin 3, granulocytemacrophage colony-stimulating factor, and interleukin 5 in eosinophilic gastroenteritis. Gastroenterology 1996;110:768-774.

9 Mishra A, Hogan S, Brandt E, Rothenberg M: An etiological role for aeroallergens and eosinophils in experimental esophagitis. J Clin Invest 2001;107:83-90.

10 Baig MA, Qadir A, Rasheed J: A review of eosinophilic gastroenteritis. J Natl Med Assoc 2006;98:1616-1619.

-11 Lee CM, Changchien CS, Chen PC, Lin DY, Sheen IS, Wang CS, Tai DI, Sheen-Chen SM, Chen WJ, Wu CS: Eosinophilic gastroenteritis: 10 years experience. Am J Gastroenterol 1993;88:70-74.

$\$ 12$ Cello JP: Eosinophilic gastroenteritis - a complex disease entity. Am J Med 1979;67:1097-1104.

-13 Roufosse F, Cogan E, Goldman M: Recent advances in pathogenesis and management of hypereosinophilic syndromes. Allergy 2004;59:673-689.

14 Blackshaw AJ, Levison DA: Eosinophilic infiltrates of the gastrointestinal tract. J Clin Pathol 1986;39:1-7.

-15 Katz A, Goldman H, Grand R: Gastric mucosal biopsy in eosinophilic (allergic) gastroenteritis. Gastroenterology 1977;73:705-709.

16 Schwartz DA, Pardi DS, Murray JA: Use of montelukast as steroid-sparing agent for recurrent eosinophilic gastroenteritis. Dig Dis Sci 2001;46:1787-1790.

17 Bolukbas FF, Bolukbas C, Uzunkoy A, Baba F, Horoz M, Ozturk E: A dramatic response to ketotifen in a case of eosinophilic gastroenteritis mimicking abdominal emergency. Dig Dis Sci 2004;49:1782-1785.

Table 1. Comparison of laboratory findings during previous hospitalization (1 week prior to admission), at current admission and 3 weeks after prednisone therapy

\begin{tabular}{lllll}
\hline Test & $\begin{array}{l}\text { Reference } \\
\text { range }\end{array}$ & $\begin{array}{l}\text { Previous } \\
\text { hospitalization }\end{array}$ & $\begin{array}{l}\text { Current } \\
\text { admission }\end{array}$ & $\begin{array}{l}\text { After prednisone } \\
\text { therapy }\end{array}$ \\
\hline White blood cells, cell/ $\mu \mathrm{l}$ & $4.8-10.8 \times 10^{3}$ & $11.5 \times 10^{3}$ & $13.8 \times 10^{3}$ & $8.3 \times 10^{3}$ \\
Hemoglobin, g/dl & $12-16$ & 11.1 & 11.6 & 13.4 \\
MCV, fl & $81-99$ & 86.0 & 88.9 & 84.0 \\
Platelets, cells/ $\mu \mathrm{l}$ & $160-360 \times 10^{3}$ & $246 \times 10^{3}$ & $371 \times 10^{3}$ & $230 \times 10^{3}$ \\
Absolute eosinophils, cells/ $\mu \mathrm{l}$ & $0.0-0.5 \times 10^{3}$ & $3,600 \times 10^{3}$ & $13,600 \times 10^{3}$ & $0.1 \times 10^{3}$ \\
Albumin, g/dl & $3.2-5$ & 2.3 & 2.0 & 3.0 \\
Total bilirubin, mg/dl & $0.1-1.2$ & 1.4 & 1.1 & 1.1 \\
Lipase, U/l & $20-50$ & 353 & 33 & 27 \\
AST, U/l & $5-50$ & 65 & 39 & 34 \\
ALT, U/l & $5-50$ & 59 & 36 & 29 \\
\hline
\end{tabular}




\begin{tabular}{|c|c|c|}
\hline \multirow{3}{*}{$\begin{array}{r}\text { Case Reports in } \\
\text { Gastroenterology }\end{array}$} & \multirow{2}{*}{\multicolumn{2}{|c|}{ Case Rep Gastroenterol 2013;7:293-298 }} \\
\hline & & \\
\hline & DOI: $10.1159 / 000354147$ & $\begin{array}{l}\text { (c) } 2013 \text { S. Karger AG, Basel } \\
\text { www.karger.com/crg }\end{array}$ \\
\hline
\end{tabular}

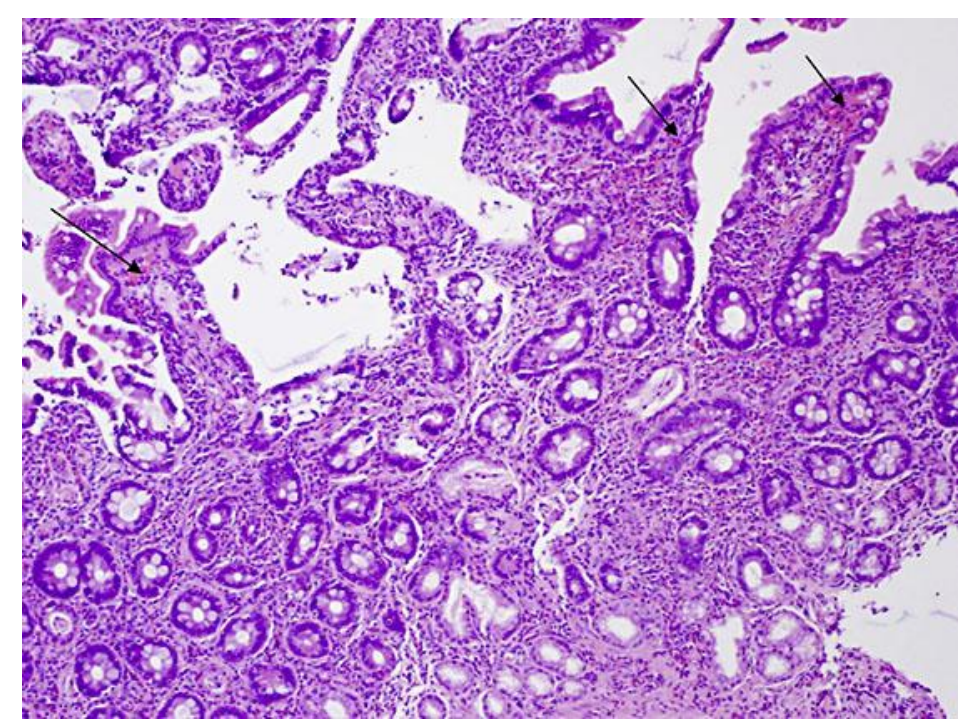

Fig. 1. Duodenal biopsy showed up to 84 eosinophils per high-power field as indicated by arrows, indicative of EGE. Hematoxylin and eosin, $\times 400$. 